\title{
Robust and Fast Assessment of Iris Image Quality
}

\author{
Zhuoshi Wei, Tieniu Tan, Zhenan Sun, and Jiali Cui \\ National Laboratory of Pattern Recognition, Institute of Automation, \\ Chinese Academy of Sciences, P.O. Box 2728, Beijing, P.R. China, 100080 \\ \{zswei, tnt, znsun, jlcui\}@nlpr.ia.ac.cn
}

\begin{abstract}
Iris recognition is one of the most reliable methods for personal identification. However, not all the iris images obtained from the device are of high quality and suitable for recognition. In this paper, a novel approach for iris image quality assessment is proposed to select clear images in the image sequence. The proposed algorithm uses three distinctive features to distinguish three kinds of poor quality images, i.e. defocus, motion blur and occlusion. Experimental results demonstrate the effectiveness of the algorithm. Clear iris images selected by our method are essential to subsequent iris recognition.
\end{abstract}

\section{Introduction}

Biometrics personal identification has been drawing extensive attention in recent years 1]. Among all the biometric traits, iris pattern has its distinctive advantages for its large inter-class and low intra-class variability. Therefore, iris recognition is no doubt a promising biometrics, which has great value in commercial and information security.

Iris image quality assessment is an important step in any iris recognition system. The existing iris recognition algorithms with good performance [2, 3, 4, are all based on certain quality images. Poor quality images will enlarge the intra-class variability and reduce the inter-class variability, and consequently increase FRR and FAR. So it is necessary to prevent poor quality images from entering subsequent processing (Fig (1).

Much attention has been paid on iris image quality assessment. Daugman [2] used a $(8 \times 8)$ convolution kernel to extract the high frequency of the image. Zhang et al. [5] used $\frac{1}{w}=\frac{\text { gradient }}{M_{i}-M_{p}}$ to measure how sharp the pupil/iris boundary is to distinguish the defocused images. Our earlier work [3] defined a descriptor $D=\left[\left(F_{1}+F_{2}+F_{3}\right), \frac{F_{2}}{F_{1}+F_{3}}\right], F_{i}=\iint_{\Omega=\left(u, v \mid f_{1}^{i}<\sqrt{u^{2}+v^{2}} \leq f_{2}^{i}\right.}|F(u, v)| d u d v$ $(i=1,2,3)$ to describe the iris image quality. However, some other differences between clear image and poor quality image are still expecting to be discovered, to make the iris recognition system faster and more robust.

To further explore this problem, an efficient iris image quality assessment algorithm is proposed in this paper. We attempt to distinguish three main cases of 


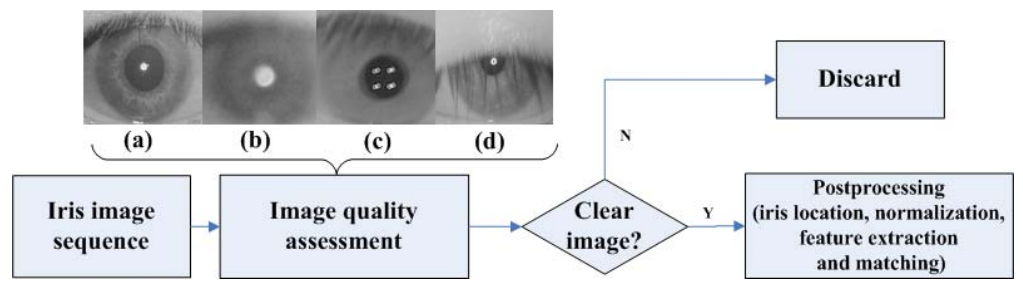

Fig. 1. Iris image quality assessment in a recognition system. (a) A clear image. (b) A defocused image. (c) A motion blurred image. (d) An occluded image.

poor quality iris images, i.e. defocus (Fig $1 \mathrm{~b}$ ), motion blur (Fig 1k) and occlusion (Fig:1d). The algorithm we proposed is conceptually simple but effective. Pupil location, which could be time-consuming, is avoided in our approach.

The remainder of this paper is organized as follows. Section 2 presents the main problems concerned. Section 3 goes into the details of our proposed method. Experimental results are provided in Section 4, and Section 5 gives the conclusion.

\section{Problem Statement}

There are three main problems concerned, i.e. defocus, motion blur and occlusion. A system that employs fixed-focus optical lens easily causes defocused iris images. Motion blurred images in our experiment are captured by a CCD sensor in interlaced scan mode, and a frame is combined by two fields with an interval of $20 \mathrm{~ms}$ or less, the resulting image involves obvious interlacing lines in the horizontal direction [3. Occluded image is the case that most area of the iris is covered by eyelid and eyelashes. It often happens if the client blinks while the images are being taken.

\section{Feature Extraction}

Our iris recognition system is supposed to assess image quality immediately after capturing the images (Fig 1). Based on such original data, no single solution can be made to separate the three kinds of images from clear images. As each of the three main problems has its own peculiarity, three features can be selected to classify them one by one. The quality of the image can be defined as $Q\left(p_{1}, p_{2}, p_{3}\right)$ :

$$
\begin{gathered}
p_{1}=\frac{1}{M \times N} \sum_{x} \sum_{y} H F P \\
p_{2}=\frac{1}{M \times N} \sum_{x} \sum_{x}^{y} V H F P \\
p_{3}=\frac{1}{M \times N} \sum_{x} \sum_{y} R O I
\end{gathered}
$$

where $M$ and $N$ are the width and height of the image respectively; $x, y$ are variables denote the pixel location; $H F P$ is the high frequency power of the image; $V H F P$ is the vertical high frequency power; $R O I$ is the Region of Interest. 


\begin{tabular}{|l|l|l|l|l|l|l|l|}
\hline-1 & -1 & -1 & -1 & -1 & -1 & -1 & -1 \\
\hline-1 & -1 & -1 & -1 & -1 & -1 & -1 & -1 \\
\hline-1 & -1 & +3 & +3 & +3 & +3 & -1 & -1 \\
\hline-1 & -1 & +3 & +3 & +3 & +3 & -1 & -1 \\
\hline-1 & -1 & +3 & +3 & +3 & +3 & -1 & -1 \\
\hline-1 & -1 & +3 & +3 & +3 & +3 & -1 & -1 \\
\hline-1 & -1 & -1 & -1 & -1 & -1 & -1 & -1 \\
\hline-1 & -1 & -1 & -1 & -1 & -1 & -1 & -1 \\
\hline
\end{tabular}

(a)

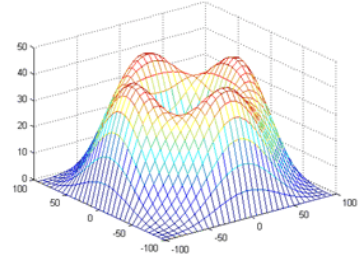

(c)

\begin{tabular}{|c|c|c|c|c|}
\hline-1 & -1 & -1 & -1 & -1 \\
\hline-1 & +2 & +2 & +2 & -1 \\
\hline-1 & +2 & 0 & +2 & -1 \\
\hline-1 & +2 & +2 & +2 & -1 \\
\hline-1 & -1 & -1 & -1 & -1 \\
\hline
\end{tabular}

(b)

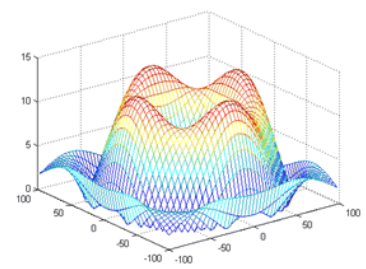

(d)

Fig. 2. (a) The $(8 \times 8)$ convolution kernel proposed by Daugman. (b) The proposed $(5 \times 5)$ convolution kernel $H_{(5 \times 5)}$. (c) The Fourier spectrum of (a). (d) The Fourier spectrum of (b).

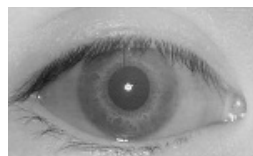

(a)

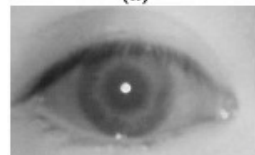

(d)

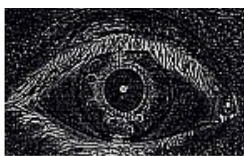

(b)

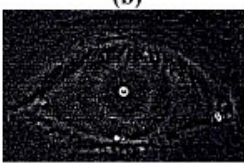

(e)

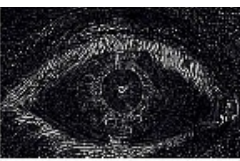

(c)

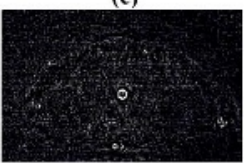

(f)

Fig. 3. (a) A clear image. (b) The result of image $a *(8 \times 8)$ kernel. (c) The result of image $a * H_{(5 \times 5)}$. (d) A defocused image. (e) The result of image $d *(8 \times 8)$ kernel. (f) The result of image $d * H_{(5 \times 5)}$.

\subsection{For Defocused Images}

A clear image has relatively uniform frequency distribution in the 2D Fourier spectrum. On the contrary, the energy of a defocused image concentrates on the lower frequency part. Therefore, using the high frequency power of the image to evaluate the degree of focus is a common method in previous research on image focus assessment.[2,6-9].

In order to obtain the high frequency power of the image, a proper high-pass convolution kernel is really important. Daugman [2] used a $(8 \times 8)$ convolution kernel (Fig 2a) to extract the high frequency power of the image. In this paper we propose a $(5 \times 5)$ high-pass filter $H_{(5 \times 5)}$, as shown in Fig 2 b.

Here we give a short analysis of this operator. It is formed by three box functions, one of size $(5 \times 5)$ with amplitude -1 , one of size $(3 \times 3)$ with amplitude 
+3 , and the last one of size $(1 \times 1)$ with amplitude -2 . The overlap of these three boxes function constitutes the operator we use. We will describe $H_{(5 \times 5)}$ in 2 aspects. One important measurement is its 2D Fourier spectrum characteristic. The proposed kernel's 2D Fourier spectrum is shown in Fig 2 d. It is a bandpass filter and its central frequency is around 0.4375 , with a bandwidth $(B W)$ of 0.3125 in which the attenuation is less than $3 \mathrm{db}$ with respect to the central frequency. Although both the proposed kernel $H_{(5 \times 5)}$ and the $(8 \times 8)$ kernel proposed by Daugman have a similar shape in 2D Fourier spectrum (Fig 2 c and $d)$ and share the same principle of filtering image, compared with $(8 \times 8)$ convolution kernel, whose passband central frequency is about 0.28125 and $B W$ is about $0.1875, H_{(5 \times 5)}$ has a higher central frequency and larger $B W$. That is to say, it can select more high frequencies. Another measurement of an operator is its efficiency. In Daugman's method, the convolution results are squared by exploiting Parseval's theorem: $\iint|I(x, y)|^{2} d x d y=\iint|F(u, v)|^{2} d u d v$, and accumulated by selecting every fourth row and fourth column [2. Doing the same work, the proposed $H_{(5 \times 5)}$ operator would take less execution time because the total multiplication is less than the $(8 \times 8)$ one. Thus we can say that $H_{(5 \times 5)}$ is computationally more efficient.

Fig 3 shows two sample images, a clear image and a defocused image. Fig $3 \mathrm{~b}$ and Fig 3 , are the images after convolution with the $(8 \times 8)$ kernel, and Fig 3 . and Fig 3 are the images after the convolution with $H_{(5 \times 5)}$. In order to get the same visual effect as the $(8 \times 8)$ one, we enlarge the image gray scale to 2.56 $((8 \times 8) /(5 \times 5))$ times. Since the $H_{(5 \times 5)}$ has a better performance in attenuating low frequencies, we can see that the clear images and defocused images become more discriminant under the proposed operator. The feature of defocus images is shown in (1).

\subsection{For Motion Blurred Images}

Motion blurred images are also undesired in iris recognition, since motion blur can severely degrade image quality. The two-field CCD sensor [12], one frame of whose image is formed by two fields, the odd field and the even field, is widely used for image capturing. The two fields are presented as adjacent rows in the resulting image. If the client moves when capturing, the two fields would present a very different scene.

As the adjacent rows are quite different in motion blurred image, the difference between every two rows of pixel is adopted as a measurement. Jarvis described SMD (Sum Modulus Difference) in his work 6] for obtaining the focus score. We find that $S M D_{x}$ which denotes the row difference is suitable for distinguishing motion blurred images, but $S M D_{x}$ is only the sum of single pixel difference. To achieve better performance, we build up an improved $(2 \times n)$ operator, the first row with amplitude -1 and the second row 1 , which is a vertical highpass filter.

In order to select a proper n, we use 200 clear and 200 motion blurred images for training. We get the threshold by using Minimal Error Criterion and finally 

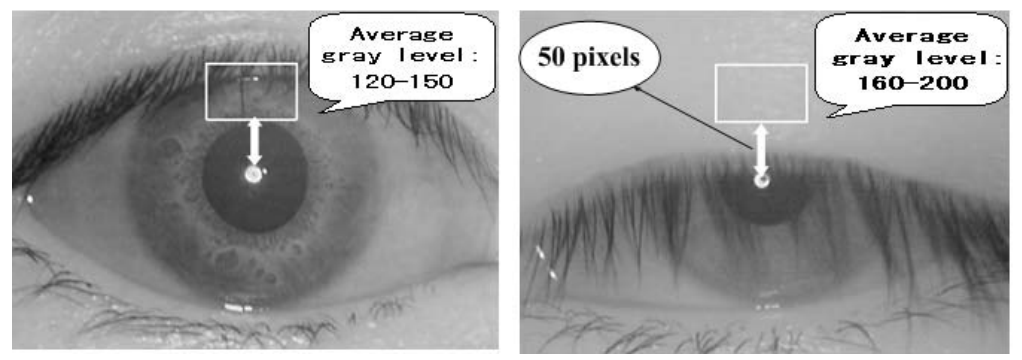

Fig. 4. Illustration of discriminating clear and occluded image

selected $n=8$ for the best tradeoff between time and performance. And the quality feature of motion blurred image is described in (2).

\subsection{For Occluded Images}

Occluded images are another big problem in iris image quality assessment. In an occluded image, at least $1 / 3$ of the iris area is covered by eyelid, and the other part is often covered by eyelashes. So, usually we can not extract accurate iris information from occluded images. That is why we consider them poor quality images and have to discard them.

The most distinct difference between clear images and occluded images is the size of iris due to occlusion. Since the radius of iris is between 100 and 120 pixels and that of pupil is between 30 and 50 pixels, given the approximate location of the pupil, then at a certain distance along the vertical direction of the pupil, we can find iris or eyelashes in a clear image, but can only find eyelid in an occluded image. Considering that the gray level of the iris and eyelid is different, we can separate the occluded images and clear images in this way. The feature for occluded image is described in (3).

\section{Experimental Results}

\subsection{Training and Testing Results}

For the purpose of validating the effectiveness of the proposed algorithm, we constructed a training dataset which contains 152 clear images, 128 defocused images, 190 motion blurred images and 105 occluded images. The testing dataset contains 300 clear images, 271 defocused images, 287 motion blurred images and 335 occluded images. The positive samples (the clear images) come from the CASIA database Version 2.0. The negative samples come from those images that can not be recognized by our recognition algorithm presented in [4].

Simply taking clear/defocus images as samples, the proposed algorithm and the algorithm proposed by Daugman, Zhang et.al. are compared in Fig [5] and Table11. The experiment is performed using Matlab 6.0 on a Pentium IV $1.3 \mathrm{GHz}$ processor with $256 \mathrm{MB}$ RAM. 


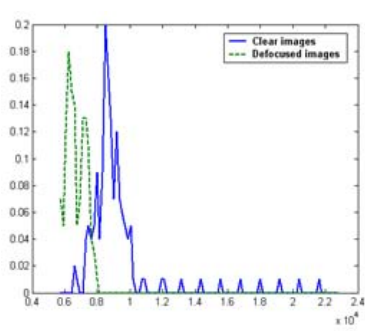

(a)

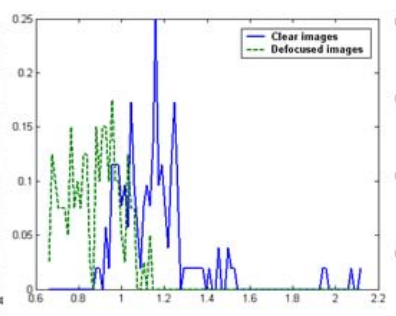

(b)

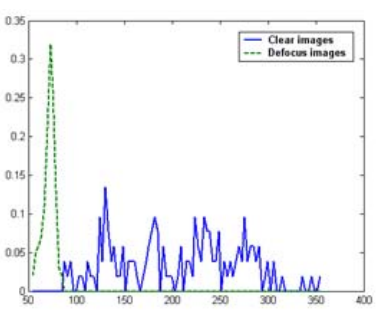

(c)

Fig. 5. Distribution of clear vs. defocused images (a)Daugman's algorithm. (b)Zhang's algorithm. (c) Proposed algorithm.

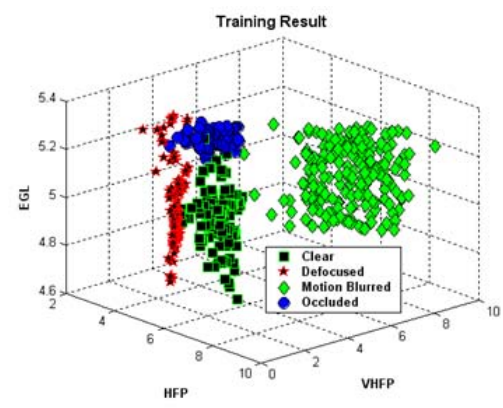

(a)

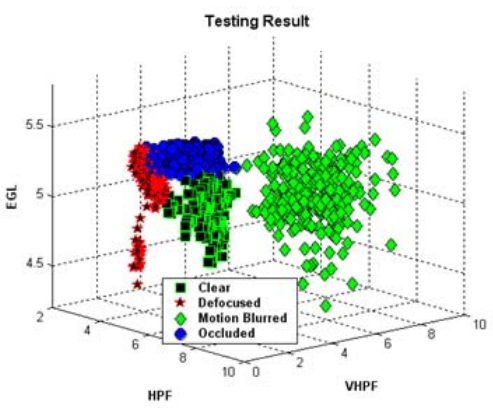

(b)

Fig. 6. (a)Training results. (b)Testing results.

Table 1. Comparison of three algorithms for detecting defocus image.

\begin{tabular}{|l|r|r|r|}
\hline $\begin{array}{l}152 \text { Clear/ } \\
128 \text { Defocus }\end{array}$ & Daugman's & Zhang's & Proposed \\
\hline time(s) & 0.1181 & 0.3431 & 0.0822 \\
\hline CCR & $92.12 \%$ & $85.62 \%$ & $98.63 \%$ \\
\hline
\end{tabular}

Table 2. Performance of previous and proposed algorithm

\begin{tabular}{|l|r|r|}
\hline & Previous & Proposed \\
\hline Training & $92.41 \%$ & $98.80 \%$ \\
\hline Testing & $89.29 \%$ & $97.68 \%$ \\
\hline
\end{tabular}

Fig [6] and Table2 illustrates the training and testing results, showing that the proposed algorithm works well. The three axes in Fig [6] respectively denote three feature components. We choose SVM to characterize the distribution boundary, for it has good classification performance in high dimensional space. Table 2 also gives the results of our previous algorithm [3].

\subsection{UBIRIS Database Assessment Results}

To further evaluate the performance of the proposed algorithm, we did another experiment on the UBIRIS database [11]. UBIRIS section1 contains 1214 images which come from 241 eyes. It is a noisy database. We take 20 clear images and 

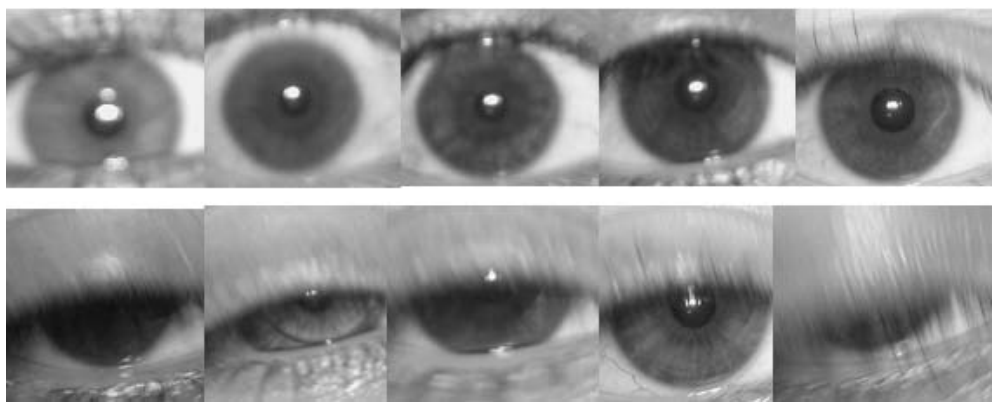

Fig. 7. Examples of poor quality images in the UBIRIS database identified by our algorithm

20 poor quality images as training samples, and the remaining images as testing samples. The results (shown in Fig.7) imply that the proposed algorithm can correctly identify the poor quality images.

\subsection{Discussions}

From the above analysis and results, a number of conclusions can be drawn as follows:

1. In detecting defocused image, Table 1 shows that the proposed algorithm is superior to the other two algorithms in terms of speed and accuracy.

2. The proposed algorithm has some distinct advantages compared with our previous algorithm. Experimental results demonstrated this conclusion. Pupil location is avoided in the proposed algorithm, which fits for a real-time recognition system.

\section{Conclusion}

Iris image quality assessment is an important step that can never be neglected in iris recognition systems. In this paper, a novel algorithm for iris image quality assessment has been proposed, which used three features to discriminate defocused, motion blurred and occluded images. It is robust and fast because we selected simple and stable features. By this method, clear images can be well selected for subsequent recognition process, and thus the intra-class distance can be reduced and the inter-class distance can be increased. It can help to further improve the performance of subsequent iris recognition and reduce the FRR.

\section{Acknowledgement}

This work is funded by research grants from the National Basic Research Program (Grant No. 2004CB318110), the Natural Science Foundation of China (Grant No. 60335010, 60121302, 60275003, 60332010, 69825105) and the Chinese Academy of Sciences. 


\section{References}

[1] Anil K. Jain, R.M. Bolle, and S. Pankanti.Biometrics: Personal Identification in Networked Society, Norwell, MA: Kluwer, (1999)

[2] J. Daugman. How Iris Recognition Works, IEEE Trans. on Circuits and Systems for Video Technology, vol. 14, no.1 pp. 21-30, (2004)

[3] L. Ma, T. Tan, Y. Wang, D. Zhang. Personal Identification Based on Iris Texture Analysis, IEEE Trans. on Pattern Analysis Machine Intelligence, vol. 25, no.12 pp. 1519-1533, (2003)

[4] Zhenan Sun, T. Tan, Y Wang, Robust Encoding of Local Ordinal Measures: A General Framework of Iris Recognition, ECCV Workshop on Biometric Authentication, (2004)

[5] Zhang et al. Method of Measuring the Focus of Close-up Image of Eyes, United States Patent, No.5953440, (1999)

[6] R. A. Jarvis, Focus Optimization Criteria for Computer Image Processing, Microsope, vol.24(2), pp.163-180, (1976)

[7] S.K. Nayar and Y. Nakagawa, Shape from focus, IEEE Trans. on Pattern Analysis Machine Intelligence, vol. 15, no.8 pp.824-831, (1994)

[8] E. Krotkov, Focusing, International Journal of Computer Vision, Vol. 1, No. 3, October, pp. 223-237, (1987)

[9] Byung Jun Kang, Kang Ryoung Park, A Study on Iris Image Restoration, in Proc. of International Conference on Audio- and Video-based Biometric Person Authentication 2005

[10] CASIA database, http://www.sinobiometrics.com

[11] H.Proenca and L.A. Alexandre, UBIRIS Iris Image Database, http://iris.di.ubi.pt.

[12] Yuqing He, Yangsheng Wang, T. Tan, Iris Image Capture System Design for Personal Identification, Advances in Biometric Personal Authentication, SpringerVerlag, (2004) 